\title{
Growth of a Vortex Polycrystal in Type II Superconductors
}

\author{
Paolo Moretti \\ Dipartimento di Fisica, Università "La Sapienza," Piazzale Aldo Moro 2, 00185 Roma, Italy, \\ and Center for Materials Science and Engineering, University of Edinburgh, King's Buildings, Sanderson Building, \\ Edinburgh EH93JL, United Kingdom \\ M.-Carmen Miguel \\ Departament de Física Fonamental, Facultat de Física, Universitat de Barcelona, Diagonal 647, E-08028 Barcelona, Spain
}

Michael Zaiser

Center for Materials Science and Engineering, University of Edinburgh, King's Buildings, Sanderson Building, Edinburgh EH93JL, United Kingdom

Stefano Zapperi

INFM UdR Roma 1 and SMC, Dipartimento di Fisica, Università "La Sapienza," Piazzale Aldo Moro 2, 00185 Roma, Italy (Received 15 December 2003; published 25 June 2004)

\begin{abstract}
We discuss the formation of a vortex polycrystal in type II superconductors from the competition between pinning and elastic forces. We compute the elastic energy of a deformed grain boundary, which is strongly nonlocal, and obtain the depinning stress for weak and strong pinning. Our estimates for the grain size dependence on the magnetic field strength are in good agreement with previous experiments on NbMo. Finally, we discuss the effect of thermal noise on grain growth.
\end{abstract}

PACS numbers: 74.25.Qt, 61.72.Mm

Understanding the phase diagram of high temperature superconductors is still a formidable challenge of modern condensed matter physics. Typically high $T_{c}$ materials behave in a magnetic field as type II superconductors, with further complications due to the broader phase space - in terms of temperature $T$ and field $H$-in comparison to conventional superconductors [1-3]. Raising the temperature, the Abrikosov vortex lattice [4] melts into a liquid [5-7], while quenched disorder leads to more complex phases such as vortex or Bose glasses [8,9].

The Bitter decoration technique is a powerful method to investigate the geometrical and topological properties of vortex matter by direct imaging [10] and provided the first direct evidence of the vortex lattice [11] predicted by Abrikosov [4]. The lattice structure is often observed to coexist with topological defects, such as isolated dislocations, dislocation dipoles, and grain boundaries. These last extended defects are the signature of a vortex polycrystal with crystalline grains of different orientations $[10,12]$. Vortex polycrystals have been observed, after field cooling, in various superconducting materials such as $\mathrm{NbMo}[10,12], \mathrm{NbSe}_{2}$ [13-16], $\mathrm{Bi}_{2} \mathrm{Sr}_{2} \mathrm{CaCu}_{2} \mathrm{O}_{8+\delta}$ [17], and $\mathrm{YBa}_{2} \mathrm{Cu}_{3} \mathrm{O}_{7-\delta}$ [18]. The grain size is typically found to grow with applied magnetic field $[12,13]$. Moreover, two-sided decoration experiments show that the grain boundaries thread the sample from top to bottom $[13,14]$, i.e., one observes a columnar grain structure. Despite the wealth of experimental observations, there is no detailed theory of vortex polycrystals. The issue is particularly interesting since recent experiments indicate that the reentrant disordered vortex phase of $\mathrm{NbSe}_{2}$, commonly believed to be amorphous, is instead polycrystalline [16].

The theoretical description of vortex matter in high $T_{c}$ superconductors is centered on the role of quenched disorder. Early theoretical considerations seemed to imply that even a small amount of disorder would lead to the loss of long-range order [19] and to the formation of an amorphous vortex glass phase [8]. Experimental observations did not confirm this view, since ordered vortex structures are typically observed even in the presence of disorder. This contradiction was resolved by a more detailed theoretical analysis of the weak disorder limit, showing a topologically ordered, long-range correlated phase, termed the Bragg glass [20]. While the presence of a Bragg glass has been confirmed experimentally [21], the precise nature of the transitions into the amorphous and liquid phases is still debated. Recent theories highlight the importance of dislocations as mediators of the transition [22], in contrast with more traditional melting theories, based on the Lindeman criterium [1]. The properties of dislocations in the vortex lattice have been the object of extensive theoretical investigations [23-26], but less is known about grain boundaries.

Here we address the problem of the formation of a vortex polycrystal from the point of view of grain growth. In field cooling experiments, magnetic flux is already present in the sample as it is quenched in the mixed phase. Thus, it is reasonable to expect that vortices are originally disordered and, due to their mutual interactions, undergo a local ordering process. Dislocation lines organize into boundaries separating grains of different 
orientation (see Fig. 1), and grain growth proceeds by grain boundary (GB) motion. We therefore analyze the dynamics of grain boundary GB in vortex matter and, in particular, their pinning by quenched disorder, which may effectively delimit the grain size.

The elastic energy of the vortex lattice can be expressed in terms of the vortex displacement field $\mathbf{u}$

$$
\begin{gathered}
\mathcal{H}=\frac{1}{2} \int d \mathbf{r}\left[c_{11}(\nabla \mathbf{u})^{2}+\left(c_{11}-c_{66}\right)(\boldsymbol{\nabla} \cdot \mathbf{u})^{2}\right. \\
\left.+c_{44}\left(\partial_{z} \mathbf{u}\right)^{2}\right],
\end{gathered}
$$

where $c_{11}, c_{44}$, and $c_{66}$ are, respectively, the compression, tilt, and shear moduli, and we assume the applied field to point along the $z$ direction. In the simplest continuum and nondispersive approximation, the elastic moduli can be estimated as $c_{11} \simeq c_{44} \simeq B^{2} / 4 \pi$ and $c_{66} \simeq \Phi_{0} B /(8 \pi \lambda)^{2}$, where $B$ is the magnetic induction, $\Phi_{0}$ is the magnetic flux quantum, and $\lambda$ is the London penetration length.

We describe an extended GB as an infinite array of edge dislocations arranged regularly along the $y$ axis, with spacing $D$ and Burgers vector in the $x$ direction. Note that, due to the columnar grain structure of the vortex polycrystal, tilt and mixed boundaries which would require a more complicated description in terms of screw and edge dislocations cannot occur. To obtain the elastic response of the GB to small perturbations, we consider a generic deformation $\mathbf{v}=\mathbf{v}_{\mathbf{n}}(\mathbf{z}) \hat{\mathbf{x}}$ for the $n$th dislocation along its glide plane, and find the elastic displacements solving the elastic equation associated with Eq. (1) with the appropriate constraints induced by the dislocations. The GB elastic energy can then be obtained as in Ref. [25] by a suitable expansion of Eq. (1). In Fourier space, the resulting elastic energy reads

$$
\mathcal{H}_{\mathrm{GB}}=\frac{\pi b^{2}}{2 D^{2}} \sum_{G_{y}} \int \frac{d Q_{y}}{2 \pi} \int \frac{d k_{z}}{2 \pi} M\left(Q_{y}+G_{y}, k_{z}\right)|\tilde{v}|^{2},
$$

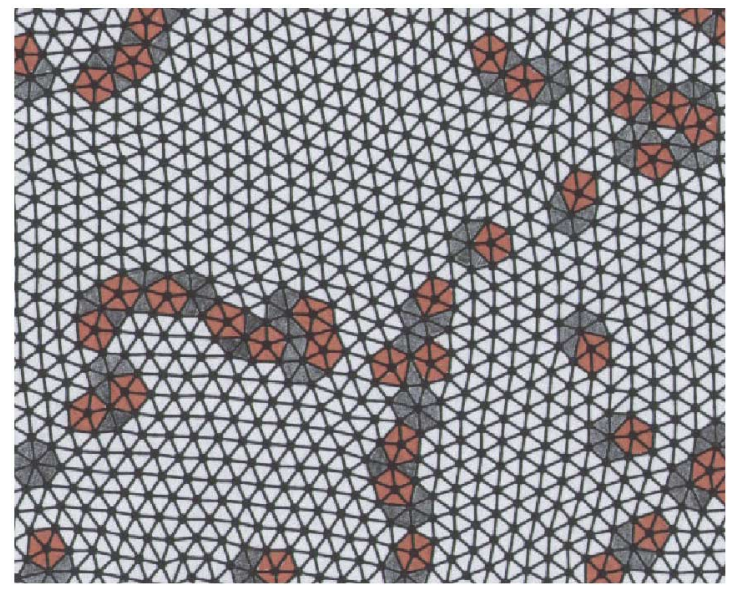

FIG. 1 (color online). Grain boundary structure from a simulation of interacting vortices after a sudden field cooling from a disordered vortex state. The colored nonsixfold coordinated vortices indicate dislocations, mostly arranged into grain boundaries. where the sum is over the reciprocal vectors $G_{y} \equiv$ $2 \pi n / D,|\tilde{\boldsymbol{v}}|^{2}=\tilde{\boldsymbol{v}}\left(Q_{y}, k_{z}\right) \tilde{\boldsymbol{v}}\left(-Q_{y},-k_{z}\right)$, and the interaction kernel is given by

$$
\begin{aligned}
& M\left(k_{y}, k_{z}\right)=2 c_{66}\left|k_{y}\right| \\
&+\frac{c_{66}^{2}}{c_{44} k_{z}^{2}}\left[\frac{\left(2 k_{y}^{2}+\frac{c_{44}}{c_{66}} k_{z}^{2}\right)^{2}}{\sqrt{k_{y}^{2}+\frac{c_{44}}{c_{66}} k_{z}^{2}}}-4 k_{y}^{2} \sqrt{k_{y}^{2}+\frac{c_{44}}{c_{11}} k_{z}^{2}}\right. \\
& \\
&\left.-2\left(\frac{c_{44}}{c_{66}}-\frac{c_{44}}{c_{11}}\right)\left|k_{y}\right| k_{z}^{2}\right]
\end{aligned}
$$

The long-distance behavior of the kernel is captured by the behavior at small wave vectors that is given by

$$
M\left(k_{y}, k_{z}\right) \simeq 2 c_{66}\left|k_{y}\right|+\sqrt{c_{44} c_{66}}\left|k_{z}\right|,
$$

corresponding to a long-range nonlocal interaction in real space. It is convenient to work in an isotropic reference frame, rescaling the $y$ coordinate by a factor $\sqrt{c_{44} / c_{66}} / 2$ [1]. The elastic kernel then becomes $M(\mathbf{k}) \simeq$ $K|\mathbf{k}|$, with $K \equiv \sqrt{c_{44} c_{66}}$. In thin films, we can neglect the deformations along $z$ and the kernel is simply given by $M(k) \simeq 2 c_{66}|k|$. Note that for an isolated vortex lattice dislocation it is correct up to a logarithmic factor to approximate the elastic energy by an effective line tension [25], but a similar procedure is not possible for a GB.

Quenched disorder induces elastic deformations of the grain boundaries and the competition between elasticity, disorder, and a driving force acting on the boundary can be analyzed in the framework of pinning theories $[1,19]$. Driving forces for GB motion can be externally induced by a current flowing in the superconductor [26] or, in our case, internally generated by the ordering process during grain growth [27]. Grain growth is driven by a reduction in energy: For an average grain size $R$ and straight GB, the characteristic energy stored per unit volume in the form of GB dislocations is of the order of $\Gamma_{0} / R$, where $\Gamma_{0}$ is the energy per unit area of a GB. Hence, the energy gain achieved by increasing the grain size by $d R$ is $\Gamma_{0} / R^{2} d R$.

Physically, the removal of GB dislocations occurs through the motion of junction points in the GB network. As junction points must drag the connecting boundary with them, which may be pinned by disorder, motion can occur only if the energy gain at least matches the dissipative work which has to be done against the pinning forces. The dissipative work per unit volume expended in moving all GB by $d R$ is $\sigma_{c} b /(D R) d R$, where $\sigma_{c}$ is the pinning force per unit area. Balancing against the energy gain yields the limit grain size,

$$
R_{g} \approx \frac{D \Gamma_{0}}{b \sigma_{c}} .
$$

In order to obtain an explicit expression for the grain size, we distinguish between weak and strong pinning regimes.

Weak pinning.-In this regime, the GB sees a random distribution of stresses $\sigma_{x y}$ due to the elastic deformations 
of the vortex lattice $[22,26]$. The random stress correlations can be obtained directly from the vortex displacement correlations in the random manifold (RM) and Bragg glass (BG) regimes [22]:

$$
\overline{\sigma_{x y}(\mathbf{r}) \sigma_{x y}(0)} \approx K^{2} \frac{a^{2}}{r^{2}} \begin{cases}\left(r / R_{a}\right)^{2 \zeta_{\mathrm{RM}}} & \mathrm{RM} \\ 1 & \mathrm{BG},\end{cases}
$$

where $R_{a}$ is the length at which the vortex displacements become of the order of the lattice spacing $a$ and corresponds to the onset of the $\mathrm{BG}$ regime. The roughness exponent in the $\mathrm{RM}$ is estimated as $\zeta_{\mathrm{RM}} \approx 1 / 5$. The pinning energy for the GB is then given by $\mathcal{H}_{\text {pin }}=$ $\sum_{n} \int d z v(n D, z) b \sigma_{x y}[v, n D, z]$. The roughness of the GB can be obtained by a scaling argument [26] comparing this expression with the elastic energy in Eq. (2), yielding $\zeta_{\mathrm{GB}}=\zeta_{\mathrm{RM}}$ in the RM regime and a logarithmic roughness for $R>R_{a}$.

The depinning stress can be computed within the framework of collective pinning theory: The energy associated with bending a grain boundary fraction of linear dimension $L<R_{a}$ over the characteristic distance $v$ can be estimated as

$$
\mathcal{E}=\frac{K b^{2}}{D^{2}} L v^{2}-\frac{K a b}{D} L v\left(\frac{L}{R_{a}}\right)^{1 / 5}+\sigma b L^{2} v / D,
$$

where the first term represents the elastic energy, the second the pinning energy, and the third the work done by an external driving stress $\sigma$ in displacing the boundary. To derive the pinning energy, we replace the sum over the dislocations forming the boundary by $L / D$, the integral over $z$ by a factor $L$, and we use the square root value of the pinning correlations. Minimizing the first two terms of Eq. (7), for $v=a \simeq b$ [26], we obtain the "plastic" Larkin length $L_{p} \simeq(b / D)^{5} R_{a}$, which is typically smaller than $R_{a}$. The depinning stress is identified as the stress necessary to depin a section of dimension $L_{p}: \sigma_{c}=$ $K b^{2} /\left(D L_{p}\right)$. Combining this expression with Eq. (5), using $\Gamma_{0} \simeq K b^{2} / D$, we obtain $R_{g} \sim R_{a}$. The identification of $R_{g}$ with $R_{a}$ was proposed in Ref. [12], but was not confirmed by experiments (see Fig. 2 and Ref. [12]). We therefore propose to interpret the experimental data under a strong pinning assumption $[28,29]$.

Strong pinning.-In this regime, pinning centers are strong and localized, and one can assume that the dislocations forming the grain boundary are pinned by individual obstacles. We consider here the case of columnar defects, oriented along the $z$ axis. This case should be relevant for the experiments of Ref. [12] where grain boundary pinning is provided by screw dislocations in the superconducting crystal. In these conditions, the problem becomes effectively two dimensional (2D) and we can directly generalize the strong pinning theory of Friedel [30], developed in the context of single dislocations, by taking into account nonlocal elastic interactions. The basic idea is to consider a GB segment as it depins from a pair of strong obstacles. The length $L$ of the seg-

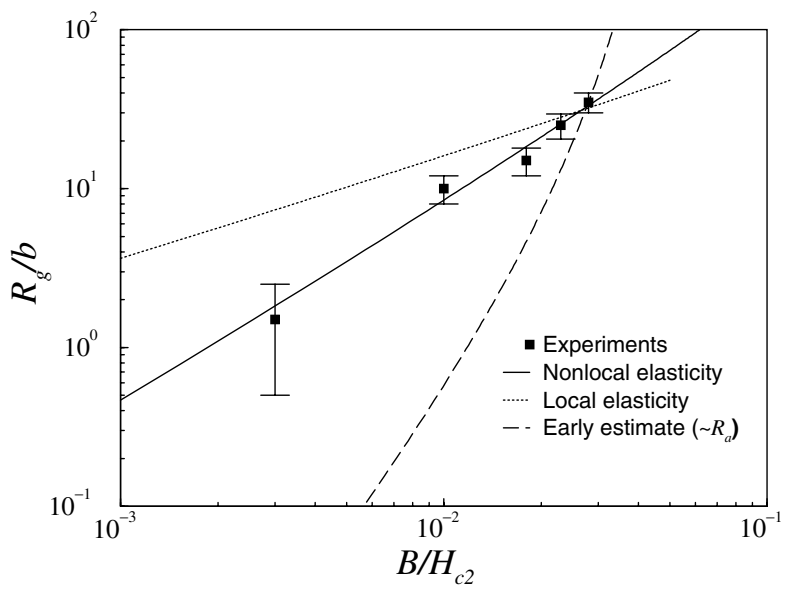

FIG. 2. The grain size of a vortex polycrystal experimentally obtained from Bitter decoration of a NbMo sample as a function of the applied magnetic field (Ref. [12]) is compared with the theoretical predictions. The calculation based on Friedel statistics with nonlocal elasticity compares favorably with the data. For comparison, we report as well the result obtained using Friedel theory with local elasticity and the earlier estimate from Ref. [12], formally equivalent to our weak pinning result.

ment corresponds to the effective spacing between obstacles along the GB, and it forms a bulge of maximum width $v$. After the GB segment overcomes the pin, it will travel by an amount which is, again, of the order of $v$ and, hence, sweep an area of the order of $L v$. At the depinning threshold, the grain boundary starts to move through a sequence of statistically equivalent configurations, and the freed segment will encounter, on average, precisely one new obstacle in the course of this process. This argument leads to the condition $L v \simeq 1 / \rho$, where $\rho$ is the area density of pinning defects. The elastic energy per unit length of the bulge of width $v$ and extension $L$ is $2 c_{66} b^{2} v^{2} / D^{2}$ (2D result), and should balance the work per unit length $\sigma b L v / D$ done by the driving stress $\sigma$ in bowing the boundary. This energy balance provides a relation between $L$ and $v$. Furthermore, at depinning the total force $d \sigma b L / D$ should be equal to the defect strength $f_{0}$, where $d$ is the sample thickness. Combining the equations above, we obtain the depinning stress $\sigma_{c} b=D f_{0} /\left(d L_{f}\right)$, where the Friedel length $L_{f}$ is given by $L_{f}=2 c_{66} b^{2} d /\left(f_{0} \rho D^{2}\right)$. Inserting the expression for the critical stress in Eq. (5) together with the scaleindependent surface tension $\Gamma_{0}=2 c_{66} b^{2} / D$, we obtain

$$
\frac{R_{g}}{b} \approx \frac{c_{66}^{2} b^{3} d^{2}}{D^{3} f_{0}^{2} \rho} .
$$

In order to use this result to fit the data in Ref. [12], we have to express it in terms of the reduced field $\tilde{B} \equiv$ $B / H_{c 2}$, where $H_{c_{2}}$ is the upper critical field of the superconductor. The field dependence is implicit in the parameters $b$ and $D$, i.e., $b \sim D \sim a \sim \tilde{B}^{-1 / 2}$, as well as in the shear modulus $c_{66} \sim \tilde{B}$ [31], and in the pinning strength 
$f_{0}$. The pinning force due to a screw dislocation was computed in Ref. [32] and is given by $f_{0} \propto \tilde{B}^{1 / 2}(1-$ $\tilde{B}) \ln \left(\xi / 2.7 b_{0} \tilde{B}\right) \approx \tilde{B}^{1 / 2} \ln \left(\xi / 2.7 b_{0} \tilde{B}\right)$, where $\xi \simeq 100 \AA$ is the coherence length [33], and $b_{0} \simeq 5 \AA$ is the Burgers vector of the screw dislocation [32]. The resulting expression predicts a linear field dependence of the grain size with logarithmic corrections. In Fig. 2, we can corroborate that the agreement of this prediction with magnetic decoration data from Ref. [12] is quite satisfactory, especially if compared to the estimate based on local elasticity assumptions.

In the discussion above, we have neglected thermal fluctuations, which could induce an activated motion of the GB, particularly in high $T_{c}$ materials. This problem can be approached generalizing scaling theories of creep for vortices and dislocations $[1,26]$. In the weak pinning regime, the energy barrier to GB motion is given by $U(\sigma)=U_{0}\left(\sigma_{c} / \sigma\right)^{\mu}$, where $\sigma$ is the applied stress, $U_{0} \simeq$ $K b^{2} R_{a}$, and $\mu=1$. In our case, the applied stress is the ordering stress, so that we have $\sigma_{c} / \sigma \simeq R / R_{a}$. Using this expression in the energy barrier for thermally activated grain growth, it follows

$$
\tau \frac{d R}{d t}=R_{a} \exp \left[-\frac{U_{0}}{k T} \frac{R}{R_{a}}\right],
$$

where $\tau$ is the appropriate characteristic time. The equation can be solved readily yielding, in the long time limit, a logarithmic growth $R(t) / R_{a}=k T / U_{0} \log (t / \tau)$. This law holds for $R>R_{a}$ when the GB would be pinned at $T=0$. In the initial growth stage $R \ll R_{a}$, we can neglect pinning forces and the dynamics is ruled by the ordering stress: $\dot{R} \sim 1 / R$, yielding a power law growth $R(t) \sim \sqrt{t}$.

In conclusion, we analyze the problem of grain growth in a vortex polycrystal, providing estimates for the average grain size that compare well with experiments on NbMo crystals. Our theory should apply to rapid field cooling experiments in which there is a competition between ordering and pinning stresses. The resulting polycrystalline structure, at least in the weak pinning regime, represents a metastable state in which the system is trapped during its evolution towards the stable Bragg glass phase. Thus, different field histories, slow cooling, or applied currents are expected to influence the resulting structure. The possibility of a thermodynamically stable polycrystalline vortex state and its possible implications for the peak effect, recently suggested by experiments $[16,34]$, still remains to be confirmed theoretically.

This work was partly supported by an Italy-Spain Integrated Action. M. C. M. acknowledges financial support from the Ministerio de Ciencia y Tecnología (Spain).

[1] G. Blatter et al., Rev. Mod. Phys. 66, 1125 (1994).

[2] E. H. Brandt, Rep. Prog. Phys. 58, 1465 (1995).
[3] T. Giamarchi and S. Bhattacharya, in High Magnetic Fields: Applications in Condensed Matter Physics and Spectroscopy, edited by C. Berthier et al. (SpringerVerlag, Berlin, 2002), p. 314.

[4] A. A. Abrikosov, Sov. Phys. JETP 5, 1174 (1957).

[5] H. Safar et al., Phys. Rev. Lett. 69, 824 (1992).

[6] F. Bouquet et al., Nature (London) 411, 448 (2001).

[7] N. Avraham et al., Nature (London) 411, 451 (2001).

[8] D. S. Fisher, M. P. A. Fisher, and D. A. Huse, Phys. Rev. B 43, 130 (1991).

[9] D. R. Nelson and V. M. Vinokur, Phys. Rev. B 61, 5917 (2000).

[10] I.V. Grigorieva, Supercond. Sci. Technol. 7, 161 (1994).

[11] U. Essmann and H. Träuble, Phys. Lett. A 24, 526 (1967).

[12] I.V. Grigorieva, Sov. Phys. JETP 69, 194 (1989).

[13] M.V. Marchevsky, Ph.D. thesis, Leiden University, 1997.

[14] M.V. Marchevsky et al., Phys. Rev. B 57, 6061 (1998).

[15] F. Pardo et al., Phys. Rev. Lett. 78, 4633 (1997)

[16] Y. Fasano et al., Phys. Rev. B 66, 020512 (2002).

[17] H. Dai, J. Liu, and M. Lieber, Phys. Rev. Lett. 72, 748 (1994).

[18] J. A. Herbsommer, G. Nieva, and J. Lurinzaga, Phys. Rev. B 62, 678 (2000).

[19] A. I. Larkin, Sov. Phys. JETP 31, 784 (1970).

[20] T. Giamarchi and P. Le Doussal, Phys. Rev. B 52, 1242 (1995).

[21] T. Klein et al., Nature (London) 413, 404 (2001).

[22] J. Kierfeld and V M. Vinokur, Phys. Rev. B 61, 14928 (2000).

[23] F. R. N. Nabarro and A. T. Quintanilha, in Dislocations in Solids, edited by F. R. N. Nabarro (North-Holland, Amsterdam, 1980), Vol. 5.

[24] E. H. Brandt, Phys. Rev. B 34, 6514 (1986).

[25] M. C. Miguel and M. Kardar, Phys. Rev. B 56, 11903 (1997).

[26] J. Kierfeld, H. Nordborg, and V. M. Vinokur, Phys. Rev. Lett. 85, 4948 (2000).

[27] P. M. Hazzledine and R. D. J. Oldershaw, Philos. Mag. A 61, 579 (1990).

[28] We have reached this result from a completely different perspective than Ref. [12], where it is assumed that above $R_{a}$ long-range order is lost in the equilibrium state, while it is now known that this is not the case [20]. Our result describes instead a metastable state due to the arrested grain growth at $T=0$.

[29] Weak pinning could still be relevant to explain the large grains observed in $\mathrm{Bi}_{2} \mathrm{Sr}_{2} \mathrm{CaCu}_{2} \mathrm{O}_{8+\delta}$ [17].

[30] J. Friedel, Dislocations (Pergamon, Oxford, 1967).

[31] Experiments are performed reasonably far apart from the transition lines to neglect any additional field and/or temperature dependence of the elastic moduli.

[32] E. Schneider, J. Low Temp. Phys. 31, 357 (1978).

[33] G. H. Kruithof, P. C. van Son, and T. M. Klapwijk, Phys. Rev. Lett. 67, 2725 (1991).

[34] M. Menghini et al., Phys. Rev. Lett. 90, 147001 (2003). 